\title{
Masque calebasse. Petite leçon guinéenne de photographie
}

A Calebasse Mask. A Little Guinean Photography Lesson

Frédéric Joulian

\section{(2) OpenEdition \\ Journals}

Édition électronique

URL : https://journals.openedition.org/tc/7929

DOI : $10.4000 /$ tc. 7929

ISSN : 1952-420X

Éditeur

Éditions de l'EHESS

\section{Édition imprimée}

Date de publication : 31 octobre 2016

Pagination : 166-177

ISBN : 9782713225291

ISSN : 0248-6016

\section{Référence électronique}

Frédéric Joulian, « Masque calebasse. Petite leçon guinéenne de photographie », Techniques \& Culture [En ligne], 65-66 | 2016, mis en ligne le 31 octobre 2018, consulté le 29 septembre 2022. URL : http:// journals.openedition.org/tc/7929; DOI : https://doi.org/10.4000/tc.7929 


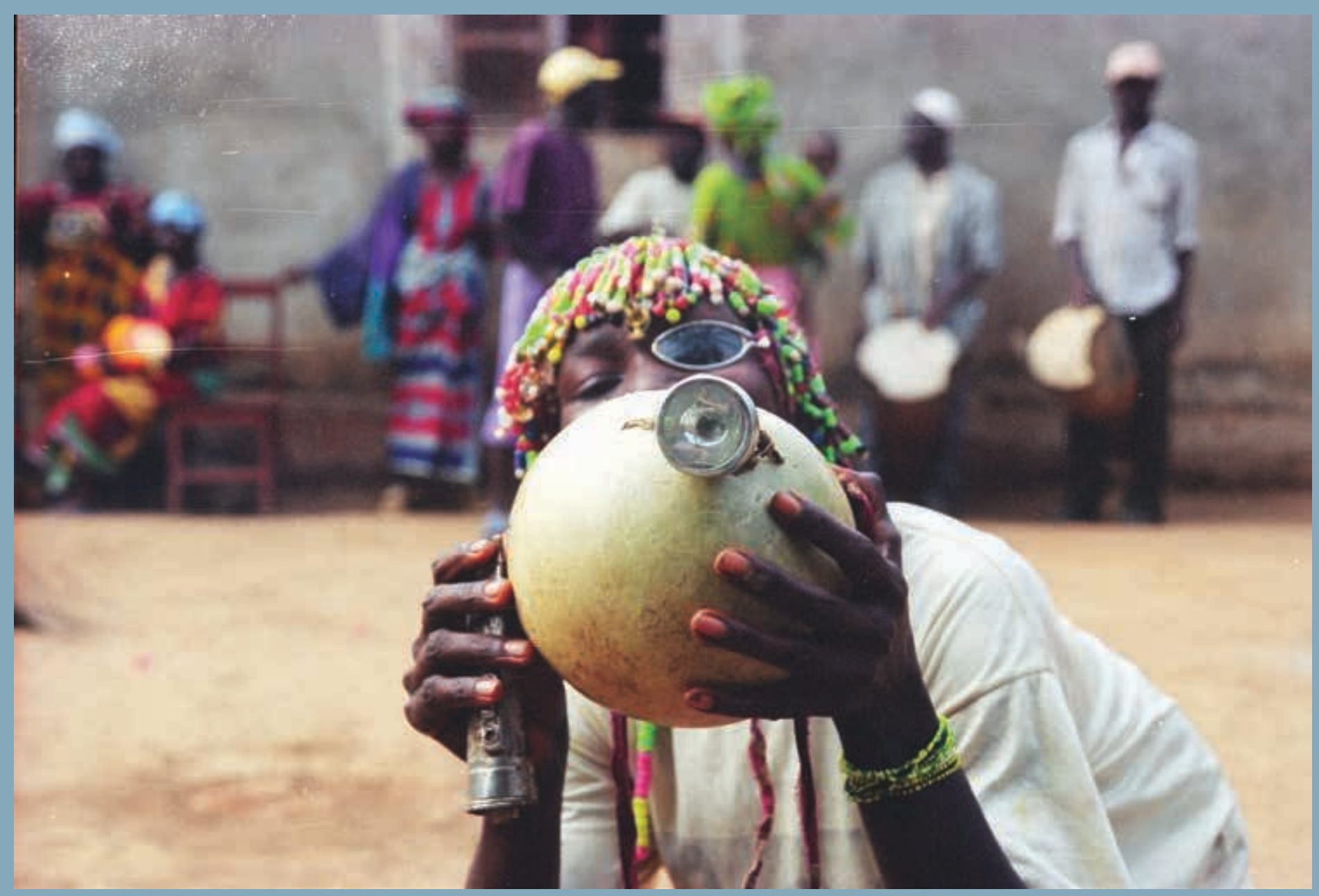




\section{Masque calebasse \\ Petite leçon guinéenne de photographie}

La série d'images présentées dans ce court essai photographique a été réalisée en avril 2005 au village de Bourounda, en Guinée littorale, à l'occasion d'une fête organisée par les villageois en remerciement de la construction d'une case destinée à un de mes doctorants d'alors, venu travailler pendant un an sur les relations des Landouma aux chimpanzés et à la nature (Leblan 2009), puis ensuite remise à la communauté villageoise.

À ma demande de voir des masques chimpanzés (demoui), connus chez les Baga de la région (Lamp 1996), une petite fête fut organisée avec un griot et un groupe de musiciens régional, spécialement invités par les villageois auxquels se joignirent un masque du village (Kosso), mais également, de façon inattendue, les femmes, principales actrices qui avaient été écartées de l'organisation.

En préparant un repas collectif pour une centaine de personnes, fabriquant des parures et colliers, mais surtout en inventant un objet original, une calebasse cassée prélevée sur un tas d'ordures ménagères, les femmes instrumentalisèrent symboliquement les invités et interférèrent dans les danses prévues par les organisateurs et les tambours. Elles s'invitèrent de façon active à la fête, détournant le spectacle et les rythmes de manière subtile et efficace, initiant à une performance politique ouverte dans laquelle d'autres acteurs vinrent se greffer inopinément.

Larène comprenait donc le masque, les musiciens et le danseur positionnés face aux visiteurs photographiant et filmant (moi-même, Mamadou Traoré, le conservateur du musée de Boké, et Vincent Leblan) auxquels, tout autour, s'ajoutèrent le reste des villageois, une ribambelle d'enfants et une dizaine de femmes qui s'immiscèrent tour à tour dans les danses.

Elles interprétèrent différentes saynètes représentant le pilage, les travaux des champs ou la pêche collective des poissons, ... évoquant aussi les traditions passées (le filage du coton notamment) faisant de cet événement le lieu d'une revendication d'existences peut-être insuffisamment prises en compte par les hommes et les chercheurs présents. 
Munie d'une calebasse, sorte d'appareil photo bricolé d'un objectif de lampe torche et d'une dragonne en pagne, remplie de dizaines de petits papiers manuscrits, la danseuse au monocle me fit face après avoir défié et photographié le danseur en action.

Au rythme des tam-tams elle percuta de façon répétée le corps de sa lampe torche sur la calebasse et s'immobilisa à quelques centimètres de mon propre objectif... me capturant dans le même mouvement. Le filmeur filmé, la situation renversée. Elle tira alors de sa calebasse magique un petit papier plié et manuscrit (un fragment de cahiers d'instituteurs déchirés) en échange de quelques pièces ou billets - usage opportun découvert rapidement au fil de l'échange.

Des déchets habilement détournés, du spectacle malmené et de la performance «enchanteresse », une interrogation et une compréhension collective émergèrent, permettant de restaurer un équilibre social, pour un temps refait.
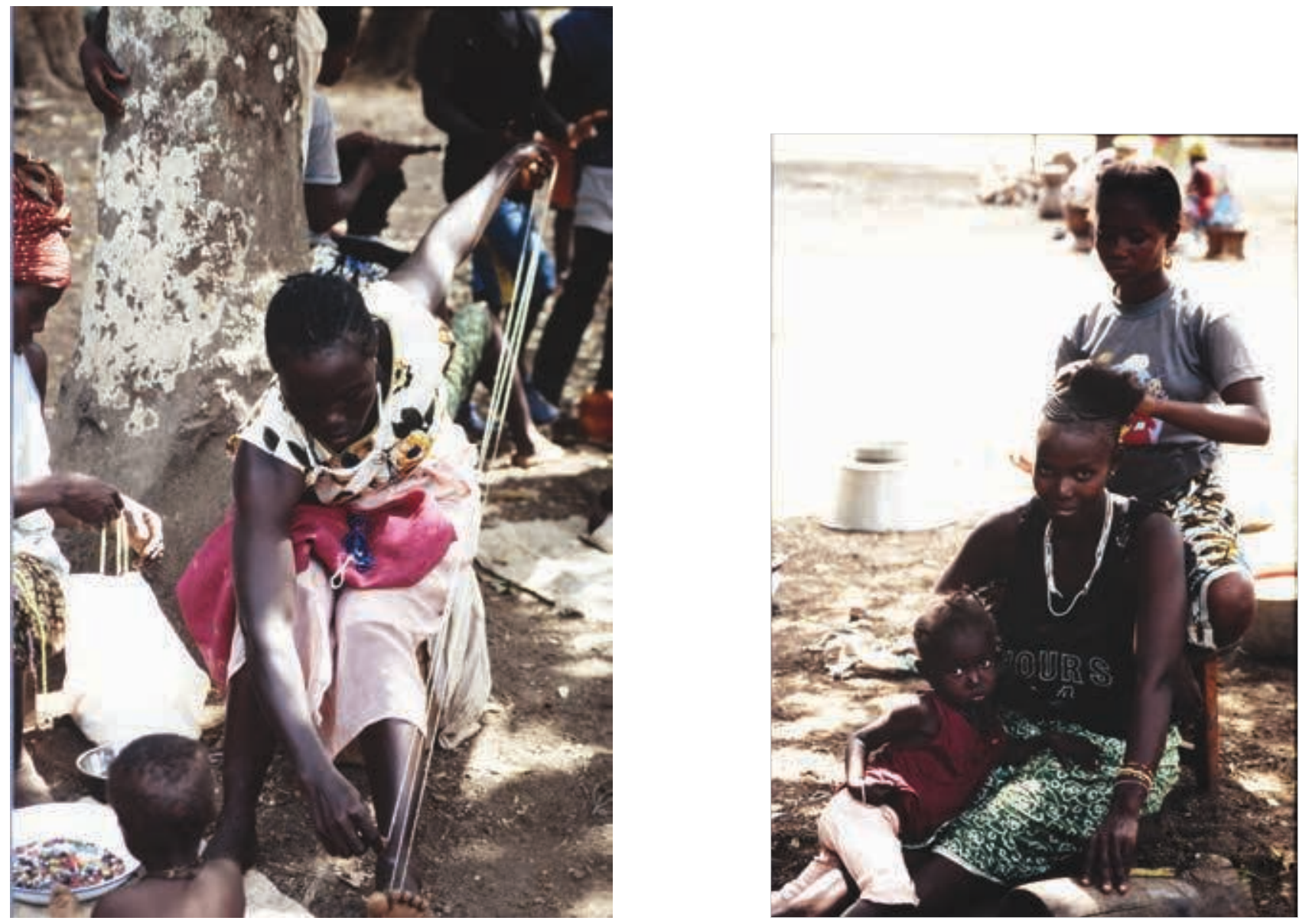

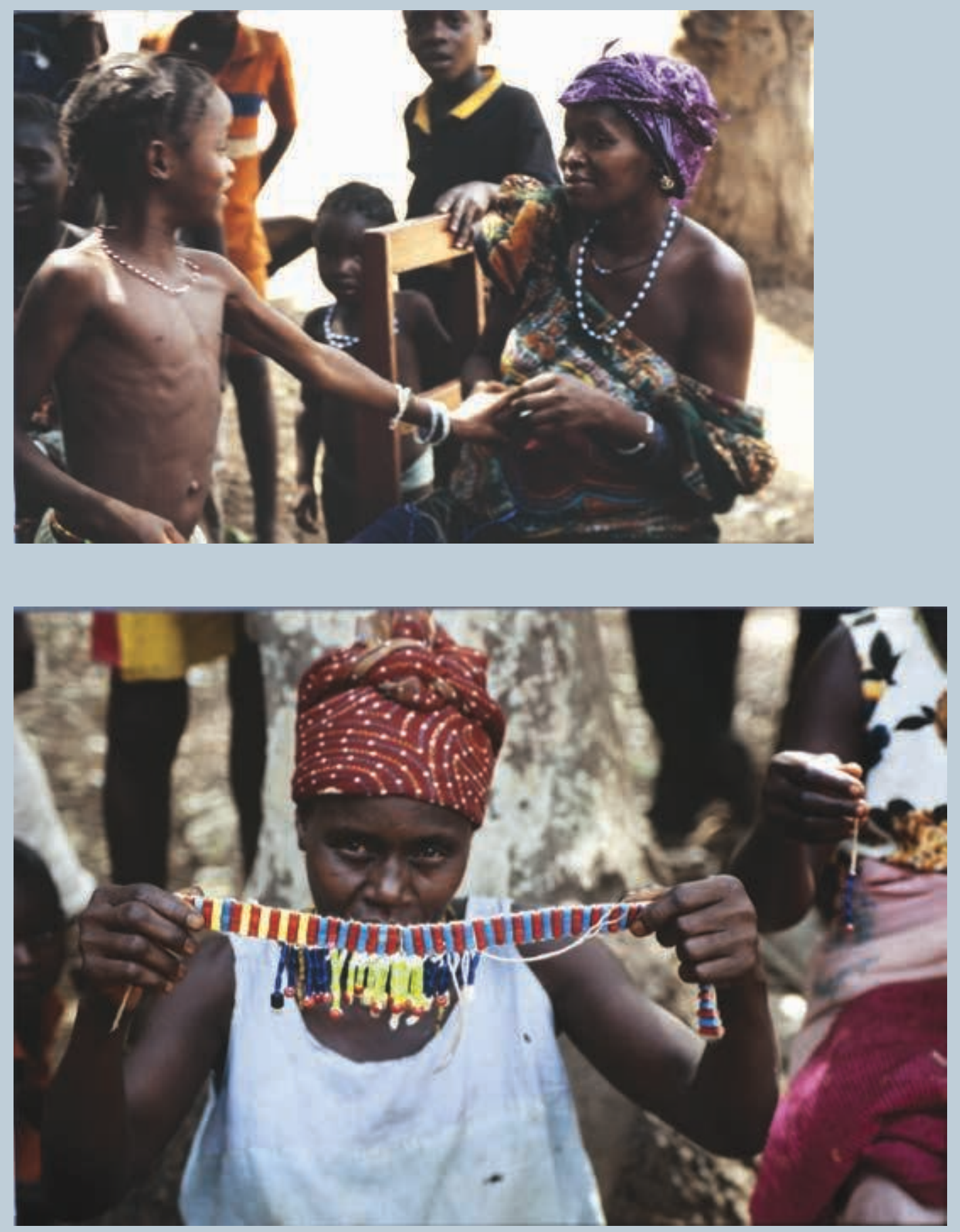

Les femmes réunissent les perles, réparent et fabriquent différents colliers et coiffes alors que d'autres continuent leurs activités et discussions... 

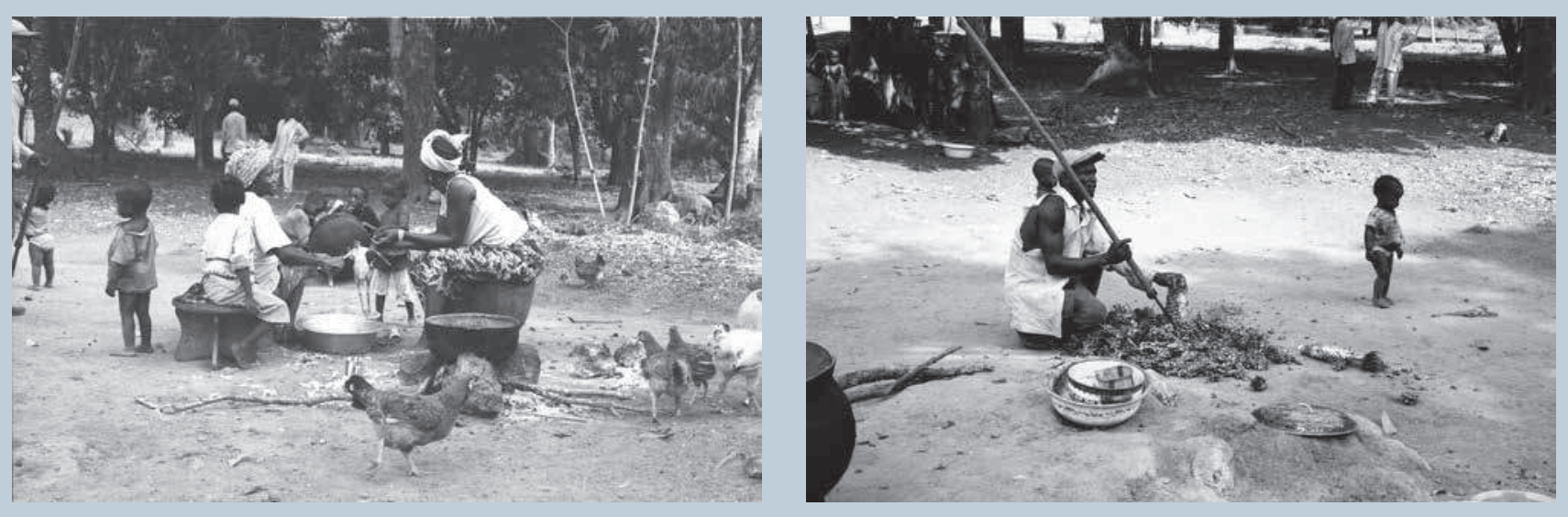

elles préparent aussi la sauce graine et les poulets alors que le griot et les musiciens commencent à tendre les peaux des tambours...

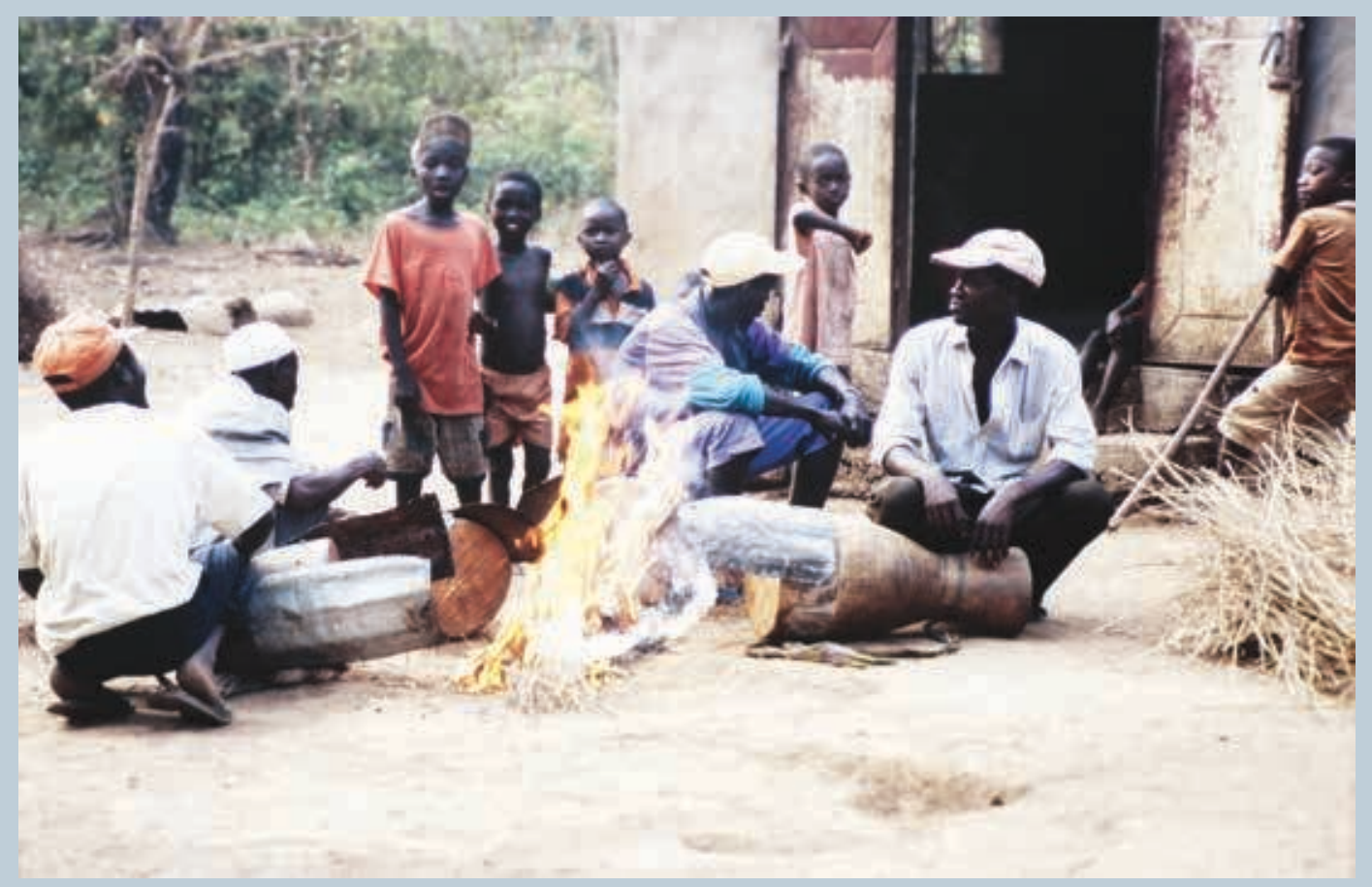




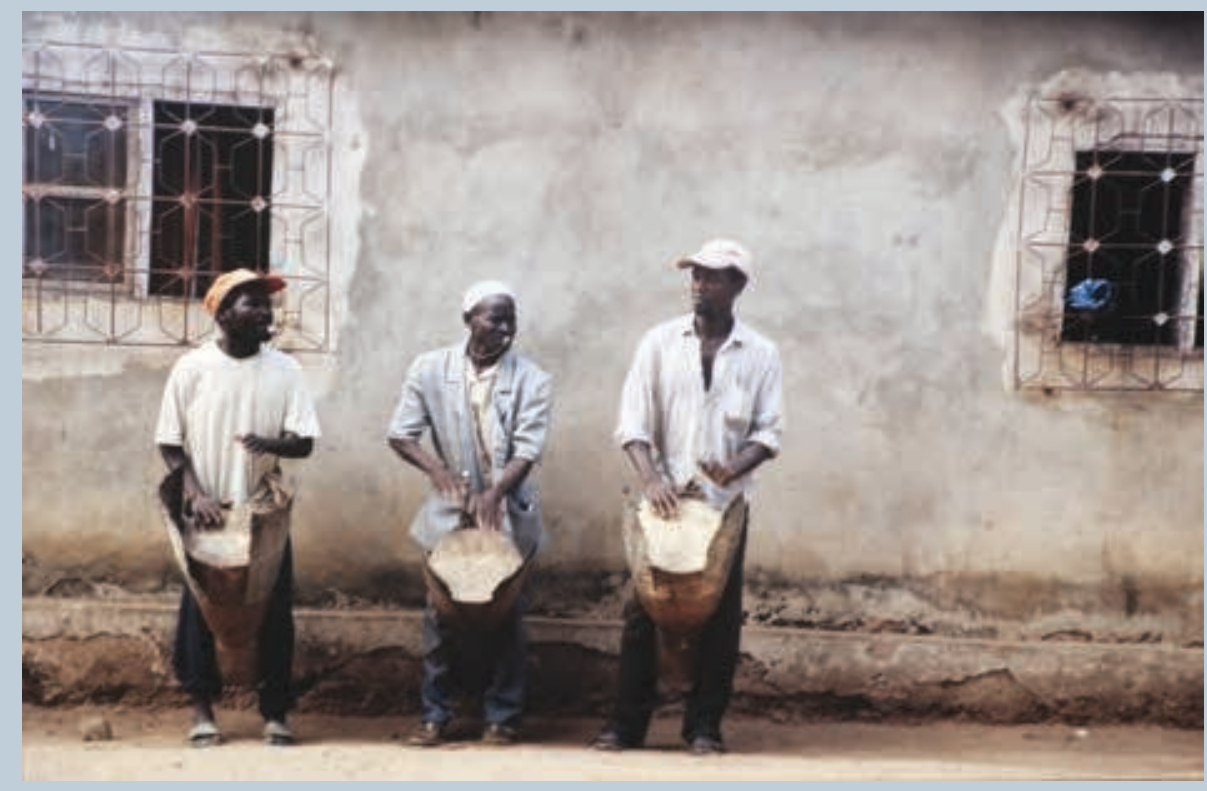

Ils trouvent rapidement le rythme qui fait sorti le masque (Kosso) de réjouissances...

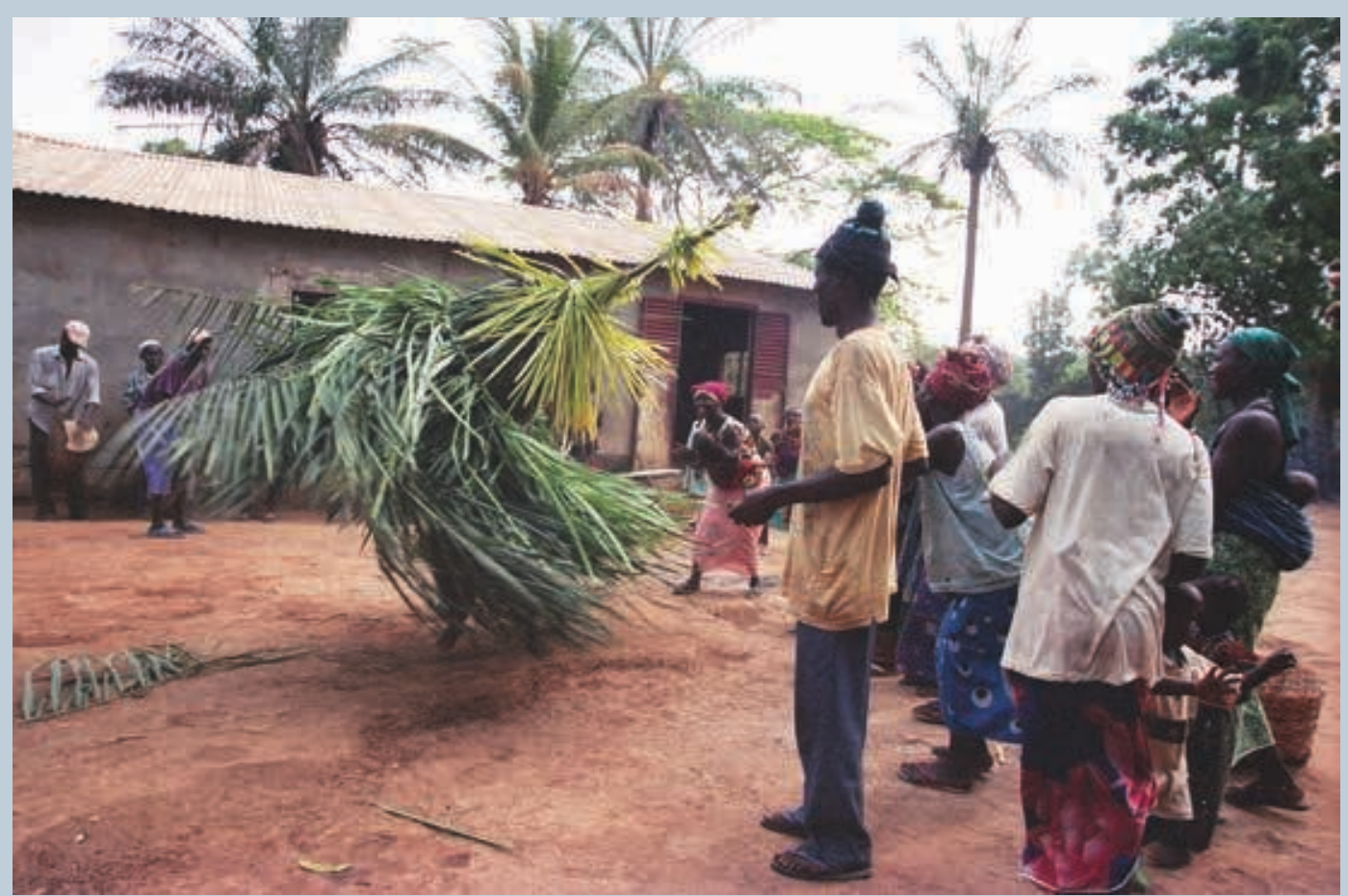




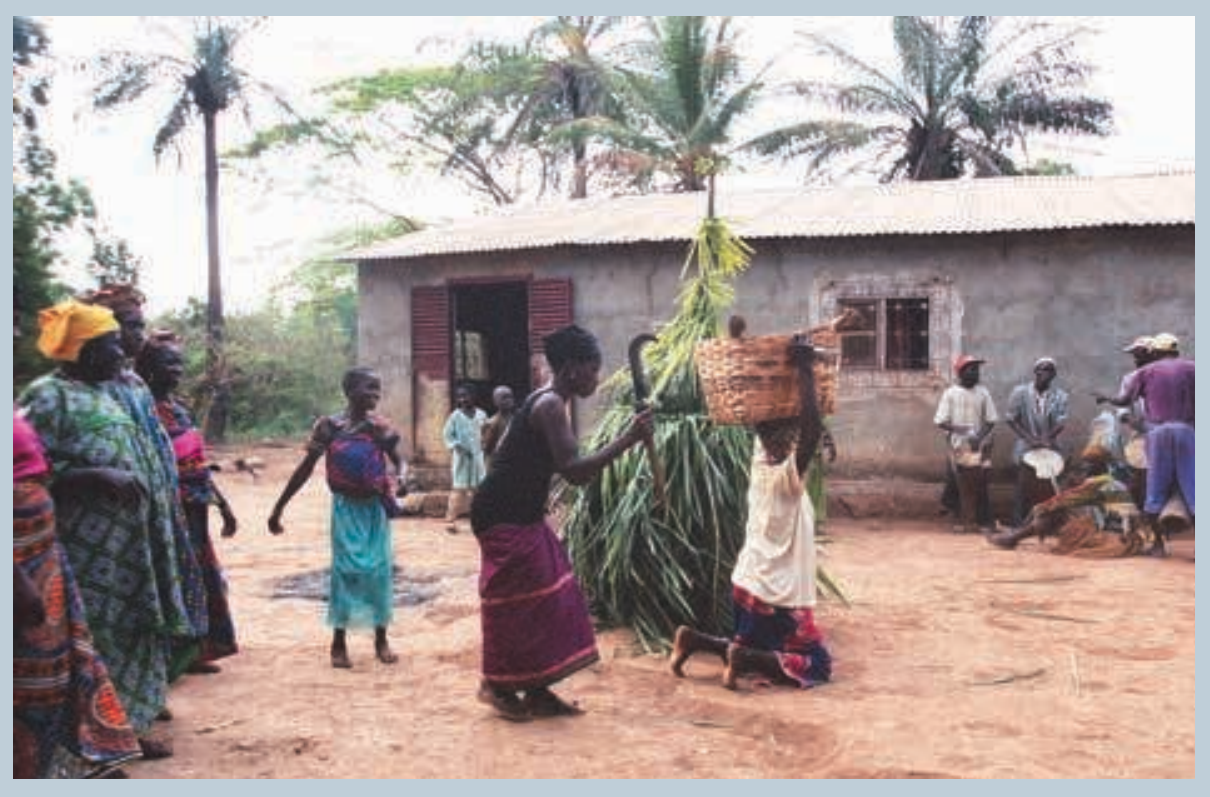

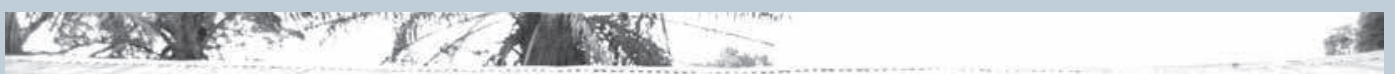

bientôt supplanté par les femmes parées qui viennent danser des saynètes traditionnelles, rivaliser entre elles, ou plus simplement prendre le rythme et occuper la place.

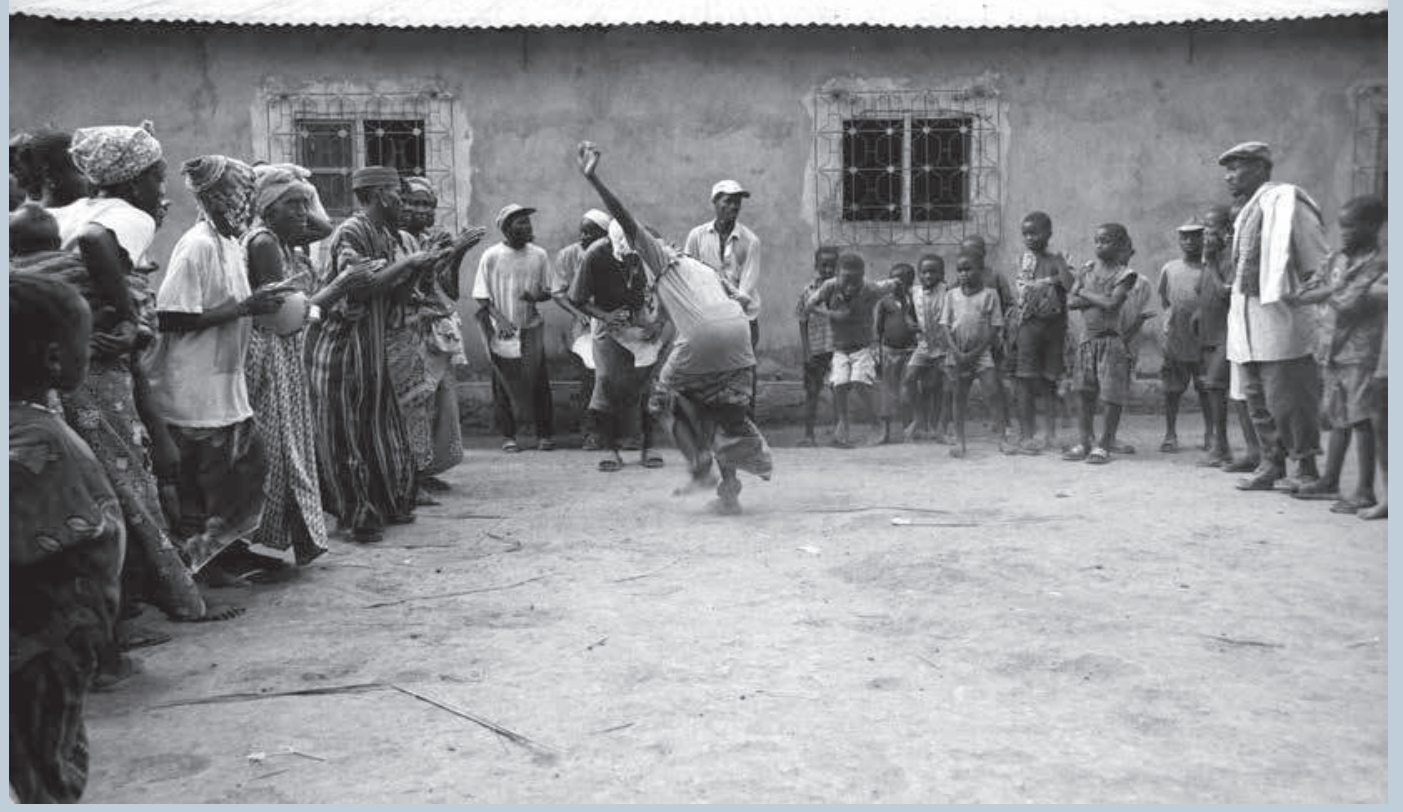




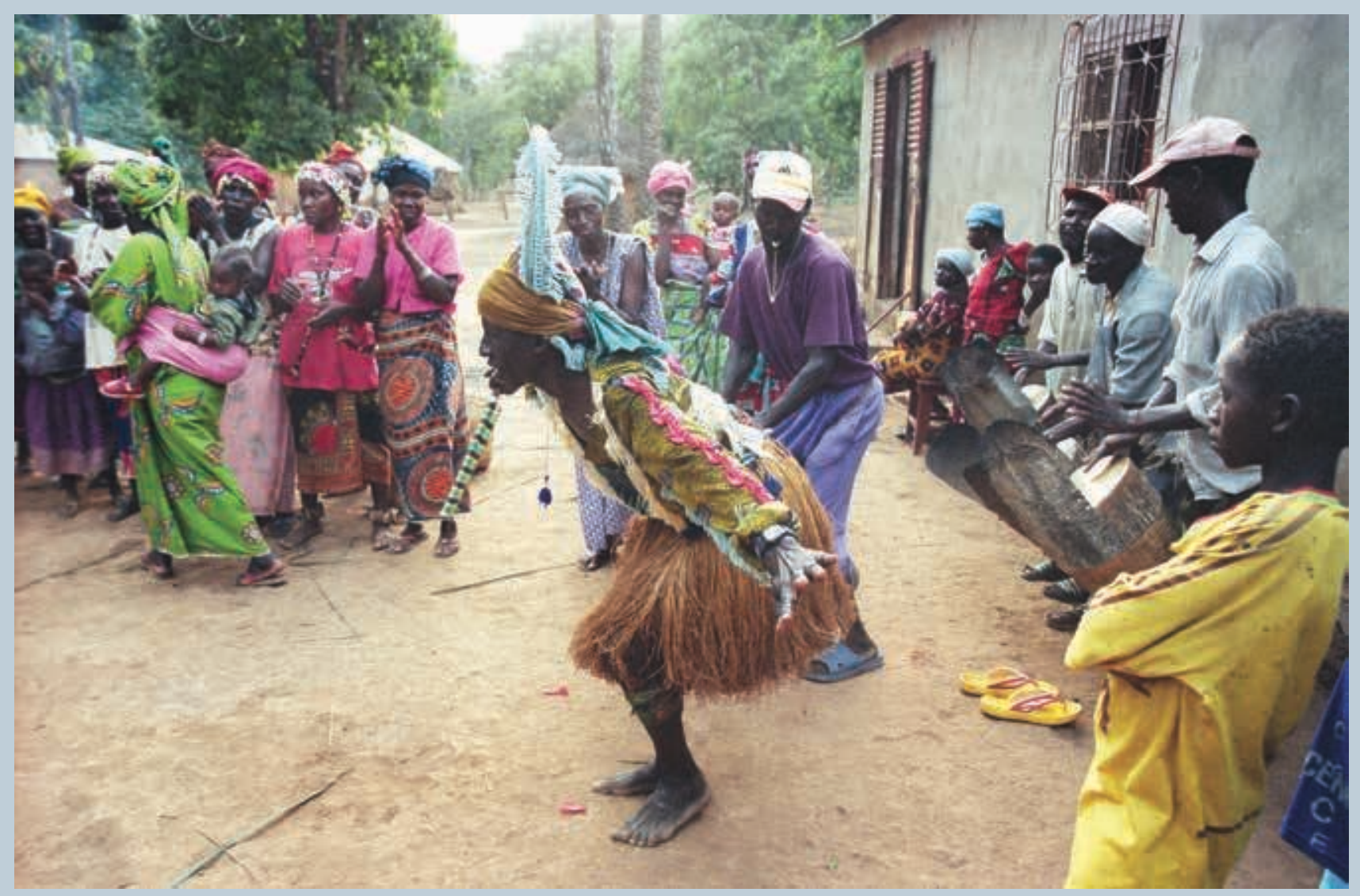

ou défier le danseur patenté du groupe. 


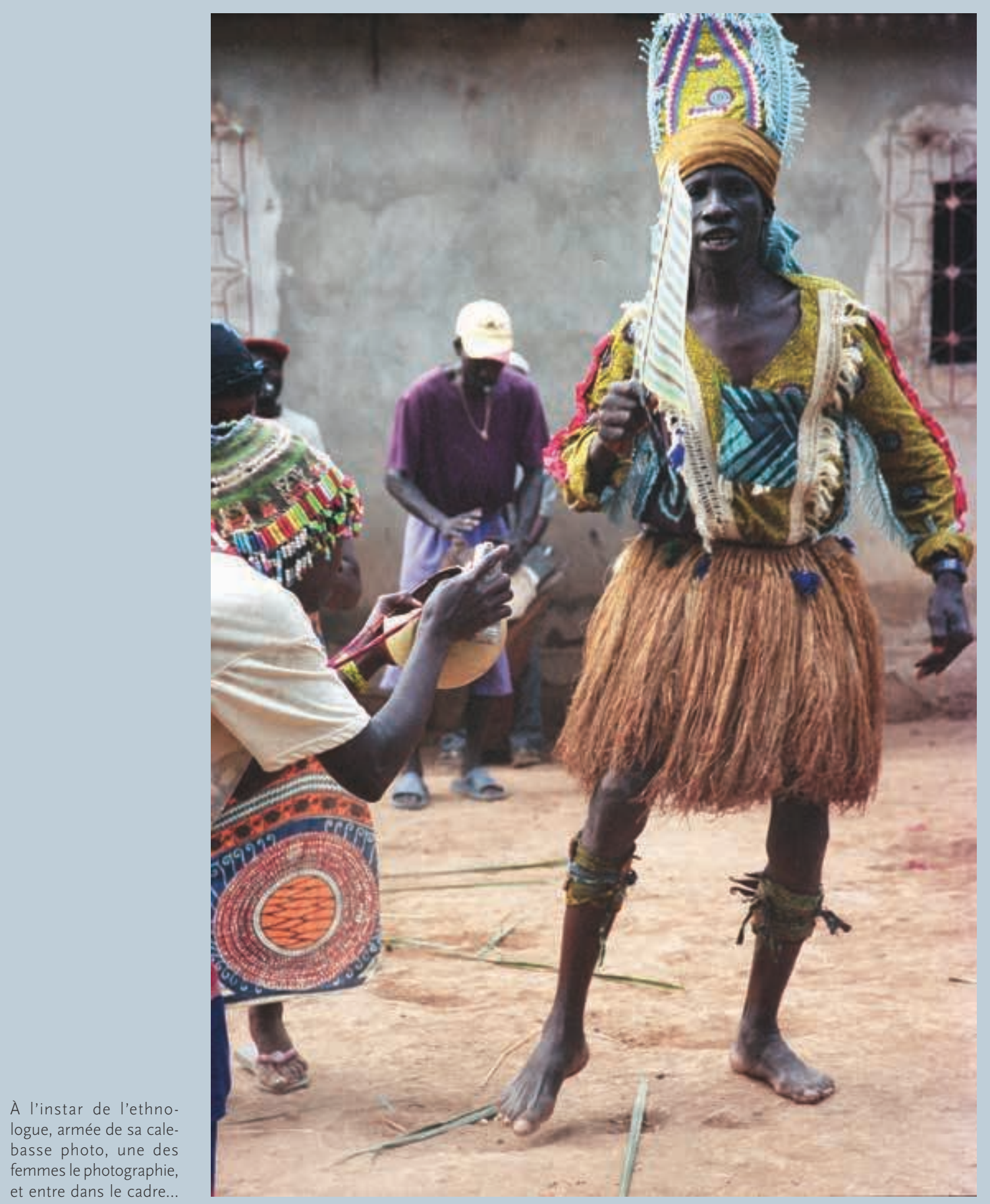



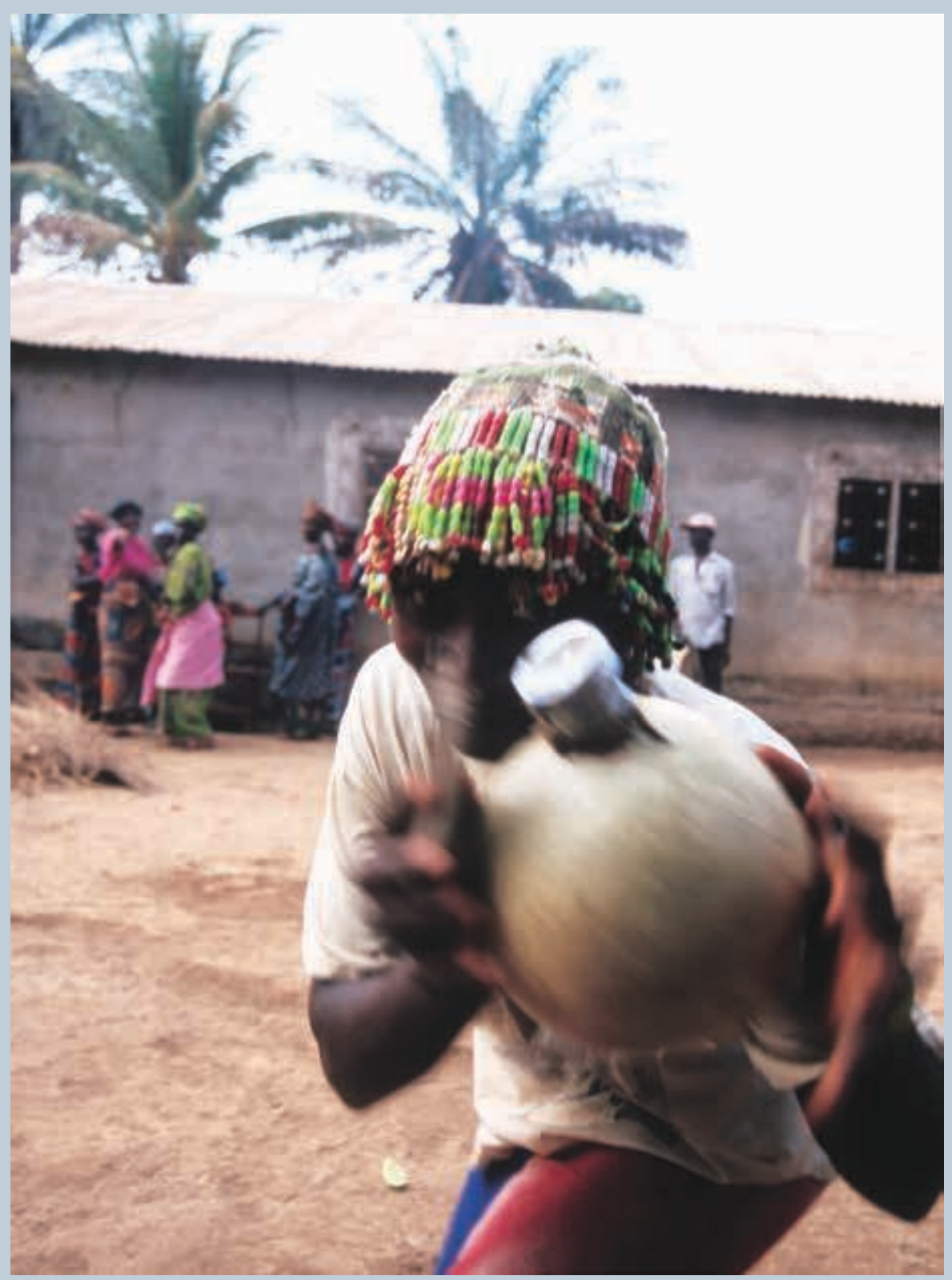

qu'elle occupe en tout sens

Elle se dirige alors vers l'observateur étranger qu'elle capture en frappant le tube vide de la torche, sorte de résonateur-obturateur figeant l'action dansée et le regard image. 


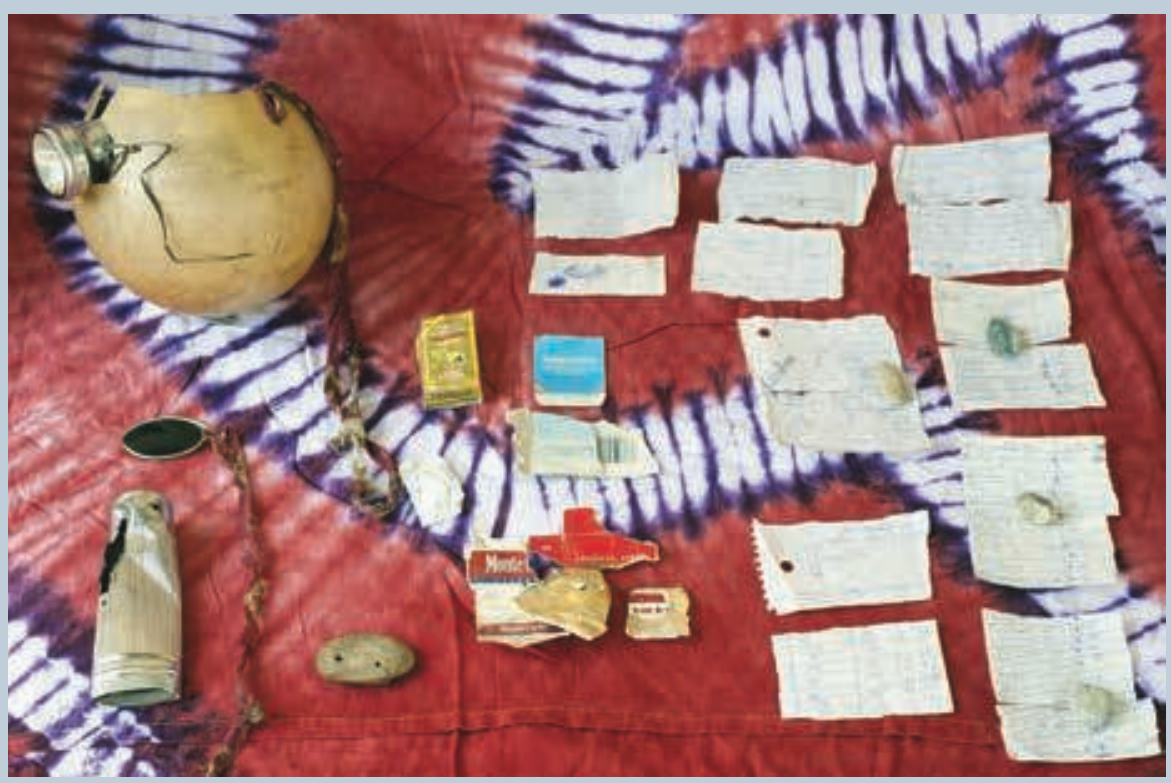

Face à mon appareil japonais, des déchets épars, la danseuse créa un objet intégrateur de la perfor mance, composé:

- d'une calebasse cassée, évidée d'un trou circu laire permettant l'installation d'une tête de lampe torche accrochée à deux trous par un ruban de tissu et une bande magnétique de cassette audio, a l'arrière par une dragonne découpée dans un morceau de pagne rouge :

- d'un verre cerclé de lunettes de soleil, ersatz de monocle attaché à un ruban pagne assorti à celu de la calebasse, tel un kit de visée dans lequel l'appareil serait donné à voir en pièces;

- du corps cassé d'une torche vidée de ses piles, instrument percutant efficace de la calebasse

- d'un ensemble d'éléments cachés contenus dans la calebasse : un grelot dans une cosse végétale avec ses graines, une petite boite de thé vert de Chine, un emballage de cube Maggi, un fragment d'emballage d'antibiotique à large spectre (Ampicilline), des fragments de boîtes de cigarettes filtres (Monte-Carlo et Gold Leaf), seize fragments de feuilles cahiers d'instituteurs (avec recommandations pédagogiques niveau CE et notes d'élèves)

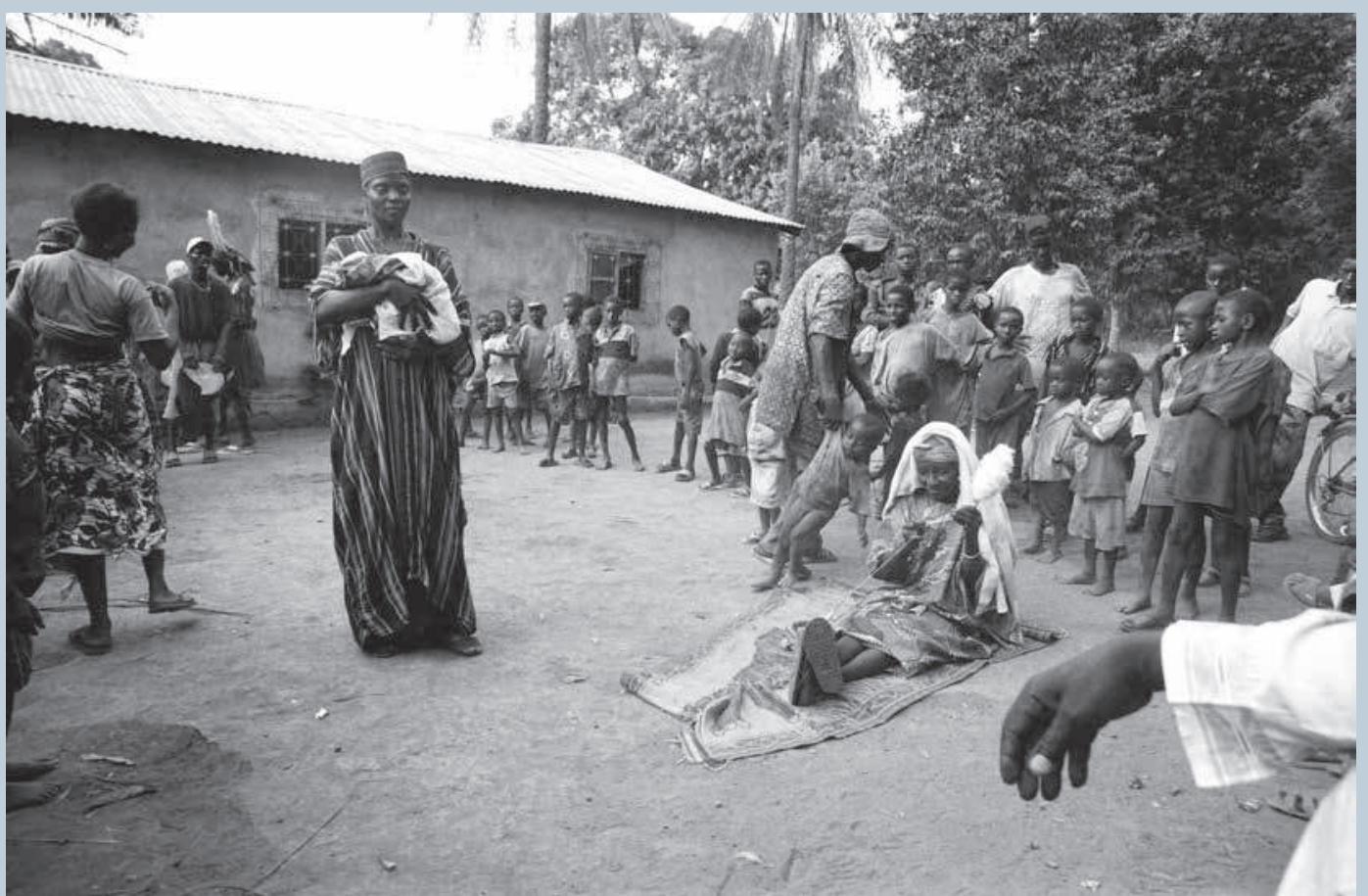

La performance publique dansée permet le détournement, l'inversion et la critique ( $c f$. Carbonnel, ce numéro), elle ouvre à de multiples registres parallèles et successifs qui vont de l'action politique immédiate aux dimensions symboliques structurantes et traditionnelles. La fête entremêle présent et passé, joue habilement devant et avec l'appareil de l'ethnologue. 


\section{I'auteur}

Frédéric Joulian, anthropologue et éthologue, Maître de conférences à l'École des hautes études en sciences sociales, a dirigé la revue TechniquesECulture de 2006 à 2016. Ses recherches portent sur les processus d'évolution et sur les significations des phénomènes techniques et culturels dans le temps long, et sur les interactions hommes-animaux en Afrique et en Europe.

\section{Iconographie}

Crédits photographiques pour l'ensemble des images: (C) F. Joulian (photographies réalisées avec un Nikon F100, pellicules Ektachrome et Kodachrome, Konika Hexar, pellicule N\&B T-MAX 135, plus une image numérique, format FX; objectifs $35 \mathrm{~mm}, 24 \times 120 \mathrm{~mm}$ et $60 \mathrm{~mm}$ ).

\section{Références}

Berliner, D. 2004 «(Re)découverte des masques Landuma (Boké, Guinée-Conakry)», ArtsECultures 5: 134-143.

Carbonnel, L. 2016 «Les rebuts captivants. Un nouvel ordre bouffon au cour de la collectivité», TechniquesÉCulture 65-66 Réparerle monde. Excès, reste et innovation: 368-383.

Lamp, F. 1996 Art of the Baga. A Drama of cultural reinvention. The Museum for African Art. Munich: Prestel-Verlag.

Leblan, V. 2009 Occupation de l'espace et utilisation de l'environnement par les chimpanzés et les humains, Ouest de la Guinée (Pays Peul et Landouma). Thèse de l'EHESS.

\section{Pour citer cet article}

Joulian, F. «Masque calebasse. Petite leçon guinéenne de photographie», TechniquesE Culture 65-66 « Réparer le monde. Excès, reste et innovation », p. 166-177.

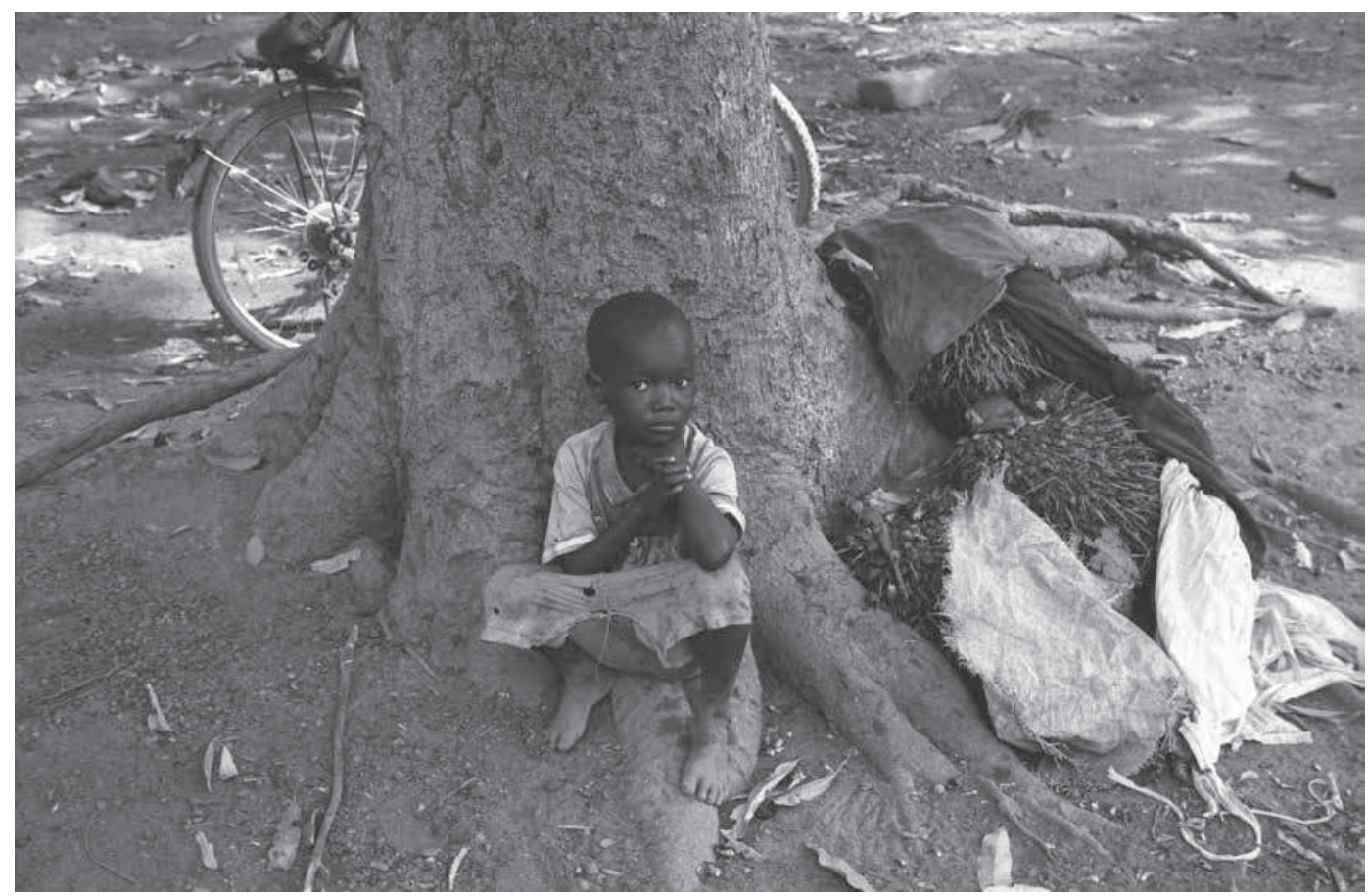

